\title{
Breakup reactions and their ambiguities
}

\author{
M. Gómez-Ramos, A. Obertelli ${ }^{\text {a }}$, Y. L. Sun \\ Institut für Kernphysik, Technische Universität Darmstadt, 64289 Darmstadt, Germany
}

Received: 15 January 2021 / Accepted: 27 March 2021 / Published online: 24 April 2021

(C) The Author(s) 2021

Communicated by Nicolas Alamanos

\begin{abstract}
We review the ambiguities in the nuclear information extracted from breakup reactions, focusing on those originating from the description of the reaction mechanism and the overall ambiguity inherent to their interpretation in terms of shell occupancies. We present the current discussion about nucleon knockout reactions and how the understanding of the reaction mechanism would help reducing uncertainties. For the former, we consider the case of ${ }^{11} \mathrm{Li}$, due to the existing large data set. For the latter, we recall the paradigmatic example of the electro-dissociation of the deuteron to address the question of the scale and scheme dependence from the theoretical framework used for the interpretation.
\end{abstract}

\section{Introduction}

Few years after the discovery and first studies of halo nuclei [1-5], Bertulani and Hussein investigated the dissociation of neutron-rich nuclei from secondary beams impinging on various targets [6]. They concluded, at that time, that the interpretation of almost all recent experimental studies with secondary radioactive beams is ambiguous. We take this statement as a starting point to address the following question thirty years later: how ambiguous is the interpretation of breakup (dissociation) reactions?

To investigate the sources of ambiguity, we review the most recent experimental work and conclusions about the structure of ${ }^{11} \mathrm{Li}$ to quantify how much various interpretations differ on the same nuclear system as discussed in Ref. [6]. We also overview the sources of ambiguities in the nuclearbreakup-reaction mechanism as they are discussed today. To illustrate the model-dependencies of orbital occupancies, we remind the historical case of the D-state probability of the deuteron and the "simplest" breakup reaction: the electrodisintegration of the deuteron.

a e-mail: aobertelli@ikp.tu-darmstadt.de (corresponding author)

\section{General description of break-up reactions}

In order to present the sources of ambiguity let us introduce a schematic expression for the T-matrix corresponding to the removal of a nucleon $N$ from the projectile $P$ leaving a core $C(P=N+C)$ via the interaction with a structureless target $T$, for simplicity:

$T=\left\langle\Psi(\mathbf{R}, \mathbf{r}, \xi)\left|V_{N T}+V_{C T}\right| e^{i K R} \Phi_{P}(\mathbf{r}, \xi)\right\rangle$,

where $\mathbf{R}$ is the coordinate between projectile and target and $\mathbf{K}$ their asymptotic initial relative momentum, $\mathbf{r}$ is the coordinate between nucleon and core and $\xi$ corresponds to all internal coordinates of the core. $\Phi_{P}$ is the wave function of the projectile and $\Psi$ is the exact final wavefunction. Usual assumptions taken in the analysis of nucleon removal reactions are to neglect exchange processes, where the emitted nucleon is not the one that interacts with the target, and to prevent the core's internal degrees of freedom from being excited during the reaction, which results in an effective $V_{C T}(\mathbf{R}, \mathbf{r})$ core target interaction that does not depend on the internal degrees of freedom of the core. This results in the wave function for the final channel $\Psi(\mathbf{R}, \mathbf{r}, \xi)$ being factorizable $\Psi(\mathbf{R}, \mathbf{r}, \xi)=\psi(\mathbf{R}, \mathbf{r}) \Phi_{C}(\xi)$ in a 3-body wave function $\psi(\mathbf{R}, \mathbf{r})$ and the wave function of the residual core $\Phi_{C}(\xi)$ :

$T \simeq \int \mathrm{d} \mathbf{R} \mathrm{d} \mathbf{r} \mathrm{d} \xi \psi^{*}(\mathbf{R}, \mathbf{r}) \Phi_{C}^{*}(\xi)\left(V_{N T}+V_{C T}\right) e^{i K R} \Phi_{P}(\mathbf{r}, \xi)$.

We note that the integral over $\xi$ only involves $\Phi_{P}(\mathbf{r}, \xi)$ and $\Phi_{C}(\xi)$ :

$\int \mathrm{d} \xi \Phi_{C}^{*}(\xi) \Phi_{P}(\mathbf{r}, \xi)=\psi_{N C}(\mathbf{r})$ 
Moreover, we can express this integral through second quantization:

$\psi_{N C}(\mathbf{r})=\sum_{\alpha}\left\langle\Phi_{C}(\xi)|a(\mathbf{r})| \Phi_{P}(\mathbf{r}, \xi)\right\rangle$,

where $a(\mathbf{r})$ is the annihilation operator for the particle of interest in a position $\mathbf{r}$, obtained from a Hamiltonian that should be consistent to that used to extract $\Phi_{C}(\xi)$ and $\Phi_{P}(\mathbf{r}, \xi)$. This allows us to recognize the definition of the spectroscopic factor

$S F=\int \mathrm{d} \mathbf{r}\left|\left\langle\Phi_{C}(\xi)|a(\mathbf{r})| \Phi_{P}(\mathbf{r}, \xi)\right\rangle\right|^{2}$

It is usual to define "single-particle spectroscopic factors" based on some effective Hamiltonian (usually a shell model) through

$S F_{\alpha}=\left|\left\langle\Phi_{C}(\xi)\left|a_{\alpha}\right| \Phi_{P}(\mathbf{r}, \xi)\right\rangle\right|^{2}$,

where $\alpha$ corresponds to a certain single-particle state. This allows to expand $\psi_{N C}(\mathbf{r})$ as a sum of normalized singleparticle wave functions $\phi_{N C}^{\alpha}$ times $\sqrt{S F_{\alpha}}$

$\psi_{N C}(\mathbf{r})=\sum_{\alpha} \sqrt{S F_{\alpha}} \phi_{N C}^{\alpha}(\mathbf{r})$

With this we can express the original T-matrix as a sum for each single-particle configuration

$$
\begin{aligned}
T & =\sum_{\alpha} \sqrt{S F_{\alpha}} T_{\alpha} \\
T_{\alpha} & =\int \mathrm{d} \mathbf{R} \mathrm{d} \mathbf{r} \psi^{*}(\mathbf{R}, \mathbf{r})\left(V_{N T}+V_{C T}\right) e^{i K R} \phi_{N C}^{\alpha}(\mathbf{r}),
\end{aligned}
$$

which translates to:

$\sigma=\sum_{\alpha} S F_{\alpha} \sigma_{\alpha}$

Note that this expression is general for any removal process in which the core can be assumed to be a spectator and shows the general procedure of separating the ingredients from nuclear structure $\left(S F_{\alpha}\right)$ and nuclear reaction $\left(\sigma_{\alpha}\right)$. It also shows that uncertainties in one of the terms directly affects the other when comparing to experiment. It also shows the dangers of inconsistency, as both $S F_{\alpha}$ and $\phi_{N C}^{\alpha}$ (included in $T_{\alpha}$ ) originate from $\psi_{N C}(\mathbf{r})$ and should be evaluated consistently. However, it is habitual to extract both terms from different prescriptions (shell model and the eigenstate of a WoodsSaxon potential, respectively), while the reaction mechanism is also derived from another Hamiltonian.
Equation 4 also allows us to consider the non-observability of the spectroscopic factors [7-9]. Indeed a unitary transformation $U(\lambda)$ produces a change in $\psi_{N C}(\mathbf{r})$ :

$\psi_{N C}(\lambda)=\sum_{\alpha}\left\langle\Psi_{C}(\xi)\left|U^{\dagger}(\lambda) a_{\alpha} U(\lambda)\right| \Phi_{P}(\mathbf{r}, \xi)\right\rangle$

which is reflected in its norm, the spectroscopic factor. It is specially interesting to remark that the evolution of operator $a_{\alpha}[10]$ :

$U(\lambda) a_{\alpha} U^{\dagger}(\lambda)=\sum_{\beta} u_{\alpha \beta}(\lambda) a_{\beta}+\sum_{\beta \gamma \delta} u_{\alpha \beta \gamma \delta}(\lambda) a_{\beta} a_{\gamma} a_{\delta}^{\dagger}+\cdots$,

which would provide an unchanging $\psi_{N C}$, clashes directly with the original assumption that the reaction process does not alter the nucleons in the core, thus establishing a connection between the ambiguities in the structure of the nucleus and the assumptions in the reaction mechanism. This poses a formidable challenge to the description of the reactions, as the mechanisms involving the excitation and de-excitation of the nucleons of the core are not well understood, and the few cases that can be tackled via ab initio calculations are very computationally expensive. It is however a general consensus that the description of the nuclear structure through standard shell-model calculations is compatible with the simple spectator-core picture assumed above, with a wide range of reactions described under this assumption. It is however questionable how quantitative these interpretations can be and if, in more extreme cases such as the removal of deeply bound nucleons, the qualitative picture still holds.

\section{The structure of ${ }^{11} \mathrm{Li}$}

An illustrative example of the ambiguities appearing in the extraction of nuclear structure information from experimental data may be found through a brief and curated, but of course sorely incomplete, story of the study of the ${ }^{11} \mathrm{Li}$ nucleus. ${ }^{11} \mathrm{Li}$ has been the subject of intensive study for more than 30 years, since the seminal work of Tanihata and collaborators [2] hinted at its halo structure through its unusually large interaction cross section. Given the large data set on ${ }^{11} \mathrm{Li}$, which have been analyzed through multiple models, generally using the factorization presented in the introduction; the current uncertainties in the description of ${ }^{11} \mathrm{Li}$ serve as an illustration of the magnitude of ambiguities found in the standard analysis of nuclear experiments.

The first experimental result we will be focusing on was published only three years later [11], and corresponds to the production cross section and the momentum distribution of ${ }^{9} \mathrm{Li}$ after the removal of two nucleons from ${ }^{11} \mathrm{Li}$ via collision 
with carbon and lead targets. The momentum distribution, obtained with the carbon target target was interpreted as the sum of two gaussians, one narrow $(\sigma=23 \mathrm{MeV} / \mathrm{c})$ and one wide $(\sigma=95 \mathrm{MeV} / \mathrm{c})$. The narrow component would originate from the removal of the two neutrons from the halo, while the wide component would be produced from neutron removal from the ${ }^{9} \mathrm{Li}$ core. The above-mentioned seminal paper by Bertulani and Hussein [6] showed that the momentum distribution, together with the dissociation cross section [12], could be explained qualitatively through two different models, one that assumed clustering of ${ }^{11} \mathrm{Li}$ into a core of ${ }^{9} \mathrm{Li}$ and a dineutron and the other that viewed the dissociation as the decay of a collective dipolar excitation in ${ }^{11} \mathrm{Li}$. The fact that such different reaction mechanisms are both able to describe the considered experimental data shows the importance of pinning down the reaction mechanism before trying to extract nuclear information from the data.

Through exploration of the $\left({ }^{11} \mathrm{Li},{ }^{9} \mathrm{Li}\right)$ reaction with multiple targets at multiple energies [13,14], it is now well accepted that the reaction takes place via direct dissociation of the two halo neutrons due to the nuclear or Coulomb potential depending on the target [15]. Multiple theoretical studies with this description of the reaction mechanism have successfully reproduced the momentum distributions and other observables [16-20]. In Fig. 1, we compare the data from [11] to an eikonal calculation which follows closely the procedure from [21], in which the formalism from Hussein and McVoy $[22,23]$ is employed to compute the stripping of the dineutron cluster, whose wavefunction with respect to the ${ }^{9} \mathrm{Li}$ core is obtained via integration of the 3-body wavefunction for ${ }^{11} \mathrm{Li}$ over the $n-n$ coordinate. The 3 -body wavefunction is computed using the P1I parametrization from [24,25] while the density for ${ }^{9} \mathrm{Li}$ and target have been taken from a Hartree-Fock calculation using the SkX effective interaction [26] and the density for the dineutron has been extracted from the 3-body wavefunction. As can be seen in Fig. 1, the calculation manages to reproduce rather well the experimental data, although it undershoots the wide component for large momenta. This is not surprising, as the used 3-body model does not allow the core to be excited and its neutrons to be emitted, while in [6] it is suggested that the wide component corresponds to the removal of neutrons from the core, though other works point to 3-body effects also influencing the wide component [16-18]. Given the rather approximate way in which the interaction between the target and the halo neutrons is treated, a better agreement perhaps should not be expected.

Even within a specific theoretical framework and when the reaction mechanism is believed to be well understood, there can be other sources of ambiguity in the analysis of experimental data. Continuing with ${ }^{11} \mathrm{Li}$, we focus now on the analysis of the ${ }^{11} \mathrm{Li}(p, p n){ }^{10} \mathrm{Li}^{*}$ reaction. The unbound nucleus ${ }^{10} \mathrm{Li}$ has been extensively studied during the years, as can

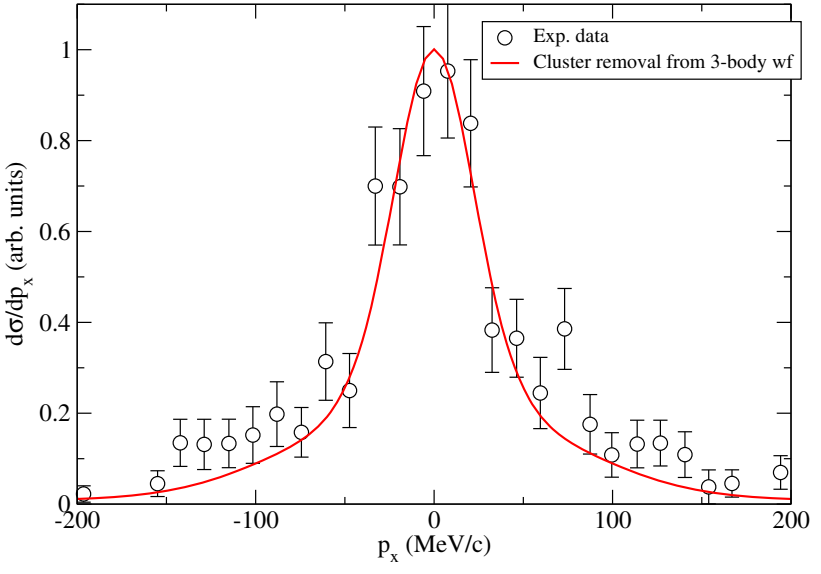

Fig. 1 Transverse momentum distribution of ${ }^{9} \mathrm{Li}$ for the reaction $\left({ }^{11} \mathrm{Li},{ }^{9} \mathrm{Li}\right)$ on a carbon target at $780 \mathrm{MeV} /$ nucleon. Experimental data are from [11] (see details in text)

be attested from Table 9.3 from [27] but there are still open questions about its structure. In [28], the ${ }^{11} \mathrm{Li}(p, p n){ }^{10} \mathrm{Li}^{*}$ reaction was measured at $280 \mathrm{MeV} /$ nucleon and the energy distribution of the residual ${ }^{10} \mathrm{Li}$ was obtained. For the analysis of the spectrum, the authors opted for an R-matrix fit, from which they obtained a $p$-wave resonance at $\sim 0.5 \mathrm{MeV}$ and a virtual state with a scattering length of $-22 \mathrm{fm}$. Because the R-matrix fit assigns arbitrary weights to each component, they could not extract the contribution of each state to the ground state of ${ }^{11} \mathrm{Li}$, though a later analysis [29] obtained relative weights between $s$ and $p$-wave by studying the profile function. However, as pointed out in [25], the energy of the different states of ${ }^{10} \mathrm{Li}$ will be correlated to their contribution to the ground state of ${ }^{11} \mathrm{Li}$, so an independent fit of the resonance energies and their magnitudes may lead to unphysical values for them. In fact, in [25], the authors presented a method where they extracted the energy distribution for different states of ${ }^{10} \mathrm{Li}^{*}$ using a three-body model for ${ }^{11} \mathrm{Li}$ [24] and the Transfer to the Continuum formalism [30] to describe the reaction process to each state of ${ }^{10} \mathrm{Li}$, assuming the ${ }^{10} \mathrm{Li}$ acted as an spectator during the reaction. Figure 2 is an adaptation of their results. P3 corresponds to a description of ${ }^{11} \mathrm{Li}$ which reproduces the energy of the $p$ wave resonance and the scattering length of the virtual state from [28] with central and spin-orbit potentials between ${ }^{9} \mathrm{Li}$ and the two neutrons. As can be seen in the top panels in the figure, this description of ${ }^{11} \mathrm{Li}$ fails to reproduce the experimental results, because the contribution of the $s$-wave virtual state is too large. Given the strong relation between the position of the virtual state and its contribution to the ${ }^{11} \mathrm{Li}$ ground state a better agreement with data requires a modification in the model for ${ }^{11} \mathrm{Li}$, not only in its parameters. In this case, the interaction P1I considered the splitting of $p$-wave resonance and $s$-wave virtual state through the coupling between the spin of ${ }^{9} \mathrm{Li}$ and the spin of the neutron in ${ }^{10} \mathrm{Li}$, while 


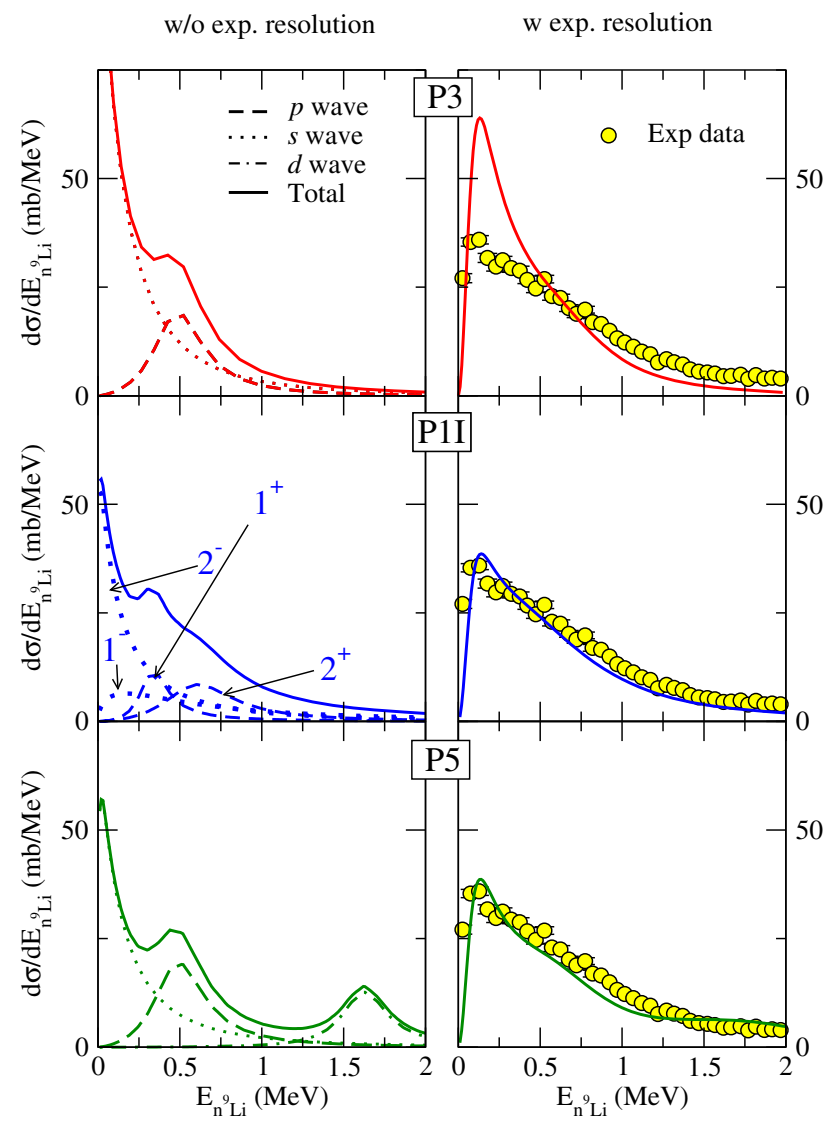

Fig. 2 Energy distribution for ${ }^{10} \mathrm{Li}$ after the ${ }^{11} \mathrm{Li}(p, p n)$ reaction at $280 \mathrm{MeV} /$ nucleon. The panels to the left correspond to calculations without folding with experimental resolution while those to the right include it. Experimental data are from [28]. Figure adapted from [25]. P3 correspond to a simple model reproducing the established energy of the $p$-wave resonance and the scattering length of the virtual state, while models P1I and P5 are extensions of this model. P1I considers splitting due to the spin-spin interaction between ${ }^{9} \mathrm{Li}$ and neutron, while P5 considers a low-energy $d$-wave resonance as suggested in [31]

P5 included a $d$-wave resonance at $1.5 \mathrm{MeV}$, as suggested in [31]. Both models manage to reproduce data remarkably well, by draining strength from the virtual state. However, this observable is not able to distinguish between them, due to the experimental resolution washing out the $d$-wave resonance. The same models have managed to reproduce the angular distribution of the ${ }^{11} \operatorname{Li}(p, d)$ reaction [24], while an analysis of the ${ }^{9} \operatorname{Li}(d, p)$ reaction seems to favour the model P1I with spin splitting over model P5 with a $d$-wave resonance [32]. This example points to possible ways to disentangle ambiguities in the analysis of experimental results: more precise measurements of the same observable and different observables that highlight different properties of the nucleus.

Turning back to the original discussion of the ${ }^{9} \mathrm{Li}$ momentum distribution, a better treatment of the three-body projectile, beyond the dineutron clustering presented in Fig. 1 would require a description of the separate interactions between the target and the three components of the projectile, considering both the absorption of one of or the two halo neutrons. Hussein published recently some works to this respect [33]. This relates to the question on whether the reaction happens sequentially (one neutron is removed or absorbed and the remaining ${ }^{10} \mathrm{Li}$ decays) or simultaneously (both neutrons are removed in the reaction). To this point, it is valuable to mention the work in [34], where the $2 n$ removal from ${ }^{14} \mathrm{Be}$ was analyzed through Dalitz plots for $\mathrm{Pb}$ and $\mathrm{C}$ targets at low energies and the signature of simultaneous breakup was found for the $\mathrm{Pb}$ target, while sequential breakup was found for the $\mathrm{C}$ target. Given the similarities between the Borromean ${ }^{14} \mathrm{Be}$ and ${ }^{11} \mathrm{Li}$ it is expectable that these results are applicable to ${ }^{11} \mathrm{Li}$. This also leads to the interesting question of the prevalence of the dineutron (spatially-correlated pair of neutrons) in the ${ }^{11} \mathrm{Li}$ halo. The study of the dineutron has not been bereft of discussions and controversies, with some works [15,3537] finding evidence against dineutron in ${ }^{11} \mathrm{Li}$ while mounting evidence during the years [38-40] points to ${ }^{11} \mathrm{Li}$ indeed presenting the spatial neutron-neutron correlations characterizing the dineutron. It should be remarked that in [38] the source of the discrepancies between different experiments was pointed out to originate from the different efficiencies of the experimental setups at low ${ }^{11} \mathrm{Li}$ energies, which shows the utter importance of adequate characterization of experimental setups when extracting information from experimental data. A very recent work to this subject has been published [41] analyzing neutron-neutron angular correlations through the ${ }^{11} \operatorname{Li}(p, p n)$ reaction, where they have found the angular correlation to be dependent on the missing momentum of the reaction, with the neutron-neutron correlations being strongest for the neutrons in the surface of ${ }^{11} \mathrm{Li}$. In this work, the composition of the ground state of ${ }^{11} \mathrm{Li}$ has been compiled from different experiments and theoretical calculations. Their compilation is presented here as Table 1 . The spread of results in terms of orbital occupancies of the two neutrons of the halo, with typical absolute variations of $\pm 10 \%$ from study to study, for $s, p$ and $d$ orbitals, and uncertainties varying from 1 to $10 \%$, shows that despite the great advances in the understanding of ${ }^{11} \mathrm{Li}$ and the study of the breakup reaction mechanism, significant uncertainties still remain. It is not surprising to find discrepancies for the occupancies, since, as seen in the previous section, the extraction of these quantities from experimental data depends on the model used in the theoretical description.

\section{Open questions on nucleon knockout reaction mechanism}

Direct reactions that knock out or transfer a single nucleon from a nucleus are a unique and powerful tool to probe singleparticle dominated states and nucleon correlations of exotic 
Table 1 Table adapted from [41]. Contribution of different neutron configurations to the g.s. of ${ }^{11} \mathrm{Li}$ obtained from various experiments and theoretical calculations

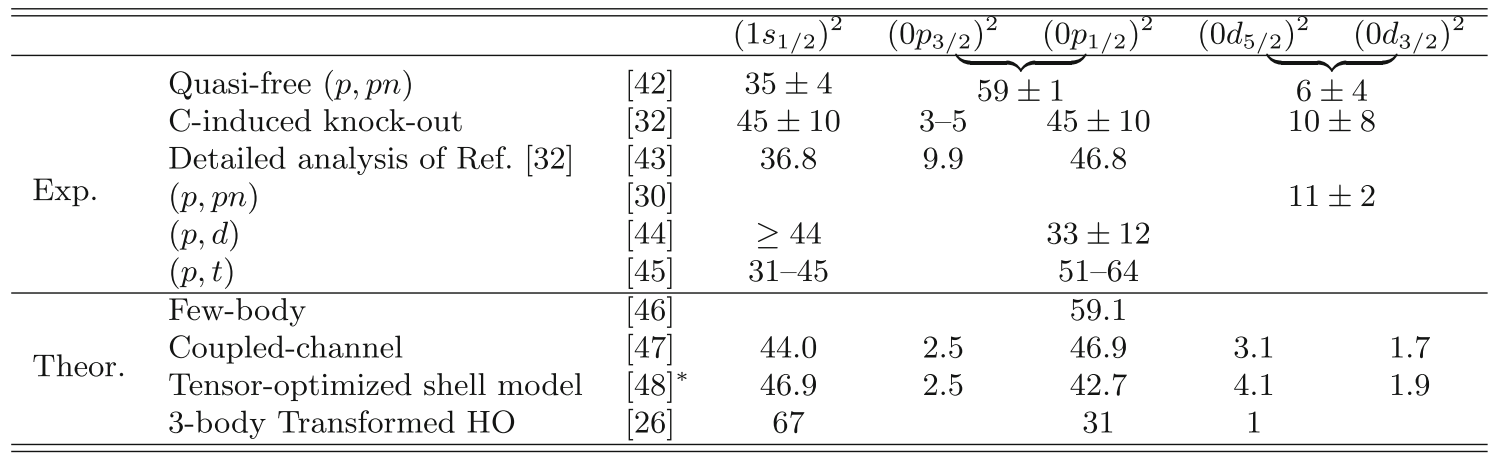

$* 0.6 \%$ and $0.5 \%$ for $\left(f_{7 / 2}\right)^{2}$ and $\left(f_{5 / 2}\right)^{2}$, respectively

nuclei $[48,49]$. The measured cross sections are often linked to the microscopic structure of the nucleus by the so-called spectroscopic factor defined in Eq. 5. Systematic studies of intermediate-energy one-nucleon removal cross sections with a $\mathrm{Be}$ or $\mathrm{C}$ target suggest that the deduced SFs based on eikonal reaction model strongly depend on the protonneutron asymmetry [50,51]. The discrepancy between experimental and predicted cross sections has been quantified by a so-called reduction factor $R_{S}$, defined as the experimentalto-theoretical cross section ratio, where the theoretical model includes both a reaction mechanism part (single-particle cross sections) and nuclear structure part (calculated SFs) as described in Sect. 2. The obtained $R_{S}$ from knockout reactions are around $0.6-0.7$ for stable nuclei, approximately consistent with those obtained with benchmark $\left(e, e^{\prime} N\right)$ reactions. For the loosely bound states, eikonal-model predictions together with shell-model spectroscopic factors are close to experimental cross sections, resulting in $R_{s}$ around unity. While for the removal of a deeply bound nucleon, the model overestimates the experimental cross section by a factor 35 , which has been first interpreted as a possible indication of missing correlations for deeply-bound nucleons in shell model calculations [50]. On the other hand, only a weak or even no dependence on proton-neutron asymmetry has been observed in transfer reactions [52-55]. Although the conclusions are based on few experimental data, still this apparent inconsistent dependence of SFs on proton-neutron asymmetry calls for a deeper understanding on the reaction mechanism and correlations in nuclei. A rather exhaustive discussion can be found in a recent dedicated review [56].

This discrepancy in the behaviour of $R_{S}$ has been attributed to failings in the applied reaction models. The adopted eikonal reaction model in the light-ion induced knockout reaction mainly has two contributions in the calculated single-particle cross section: the stripping/inelastic breakup and the diffractive/elastic breakup. The target nucleus, whose internal structure is not treated explicitly in the calcula- tion, is excited in the former and remains in the ground state in the latter. These two nucleon-removal mechanisms have been identified experimentally from their different kinematics [57]. Depending on the separation energy of the removed nucleon [58], the elastic breakup could contribute $15-35 \%$ to the one-nucleon removal cross section. In general, the eikonal model is formulated based on three approximations [22,23,59-61]: the sudden/adiabatic approximation, which ignores the internal motion of nucleons during the reaction, the eikonal approximation, which assumes the scattering nucleons follow a straight line, and the spectator-core approximation, which treats the knockout residue as a spectator that can interact at most elastically with the target. The spectator-core model was initially elucidated by Hussein and McVoy [22] to discuss the stripping process. The validation of the spectator-core assumption has been checked by AlKhalili when the core itself is loosely bound [62], where less than $10 \%$ correction for the stripping cross sections was found. However, in non-direct reaction processes, such as multiple scattering inside of the projectile, the excitation and decay of the spectator core could influence the deeply bound nucleon-removal cross section with a composite target [63]. In particular, recent theoretical and experimental works suggest that there may exist core excitation contributions that could significantly reduce the deeply bound nucleon-removal cross sections $[63,64]$. In fact, intranuclear cascade calculations, which include these effects but no microscopic information on the detailed nuclear structure, support the important role of evaporation in the cross sections for deeply bound nucleon removal from very asymmetric nuclei, i.e. leading to a fragile residual nucleus of low separation energy [63]. Still, a fully quantum mechanical and microscopic description is missing.

In addition to the knockout cross sections, the momentum of the knockout residue is often measured experimentally and is an observable which offers insights into the underlying reaction mechanism. Concerning parallel momentum distri- 


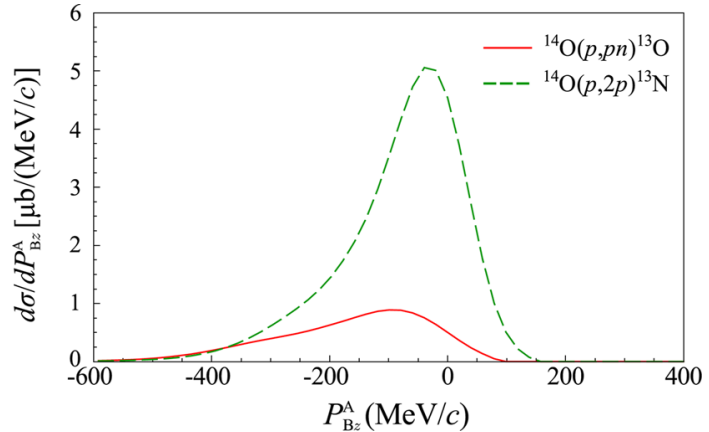

Fig. 3 Momentum distribution of ${ }^{13} \mathrm{O}$ and ${ }^{13} \mathrm{~N}$ for the ${ }^{14} \mathrm{O}(p, p n)$ reaction at $100 \mathrm{MeV} /$ nucleon. Figure adapted from Ref. [65]

butions (PMDs) from the knockout involving deeply-bound nucleons, asymmetric PMDs have been observed by several experiments [66-68], i.e., a long low-momentum tail [66-68] and an abrupt high-momentum cutoff [68], in contrast to the symmetric PMDs predicted by the eikonal model. The origin of the observed asymmetric PMDs is still not clear. The low-momentum tail in some limited-asymmetry-tail cases can be fairly well described by the fully quantum mechanical coupled discretized continuum channel (CDCC) calculations including high-order effects for the elastic breakup component $[60,69]$. Moreover, the advanced transfer to continuum method developed by Bonaccorso and collaborators [70,71], and based on a target-nucleon optical potential, can reasonably describe the recent very asymmetric PMD of $\left({ }^{14} \mathrm{O}\right.$, $\left.{ }^{13} \mathrm{O}\right)$ [68].

Proton induced quasi-free knockout $(p, p N)$ is a complementary tool to intermediate-energy heavy-ion induced one-nucleon removal reactions. The $(p, p N)$ reaction mechanism is simpler as the proton can be treated as a structureless probe, while it is expected that it probes a different part of the radial wave function compared to heavy-ioninduced nucleon-removal reactions due to a different absorption. Notably, the recently extracted SFs for the oxygen isotopic chain from quasi-free knockout reactions also show a very weak dependence on proton-neutron asymmetry $[72,73]$ as opposed to the findings in heavy-ion induced knockout reactions. Later analysis with different theoretical methods show a consistent weak dependence $[74,75]$. Similarly to the heavy-ion induced knockout reactions, the momentum distributions from $(p, p N)$ reactions are also sensitive to the orbital angular momentum of the ejected nucleon $[65,76-$ 78]. A recent DWIA (distorted-wave impulse approximation) calculation on the ${ }^{14} \mathrm{O}(p, p N)$ reaction also predicted large asymmetric PMDs [65], as shown in Fig. 3. The asymmetric PMDs were investigated in detail by the authors. The lowmomentum tail is caused by the attractive potential between the outgoing nucleons and the residue while the cutoff at high momentum side is due to the phase volume effects in the energy and momentum conservation [65], as in the case with heavy-ion targets. Experimental verification of PMD from $(p, p N)$ reactions with good resolution is still necessary which will provide a test for a reliable reaction model for SF study. In addition, exclusive measurements of the knockedout proton or neutron and the recoil proton will also provide important information to clarify the relevant processes in the quasi-free knockout mechanism $[79,80]$. Given the non-observable nature of the spectroscopic and $R_{s}$ factors, the question of model dependence arises. In the next section we present the simpler deuteron electro-disintegration reaction as a proxy to analyse the effects of model dependence.

\section{The electro-disintegration of the deuteron}

We discussed above the ambiguities in the modeling of the breakup reaction mechanism and illustrated the typical discrepancies between data and predictions. Beyond the approximations to model the reaction mechanism, the interpretation of cross sections in terms of non-observable quantities such as spectroscopic factors or shell occupancies depends on the theoretical framework. We focus here on the historical example of the electro-disintegration of the deuteron to highlight the essence of ambiguities related to the interpretation of direct-reaction data.

The deuteron has been used as a benchmark for $\mathrm{NN}$ interactions. Its wave function can be reduced to a one-dimension problem when expressed in the center of mass coordinates

$\Psi=\left(\frac{u(r)}{r} Y_{00}+\frac{w(r)}{r} Y_{2 m}\right) \chi_{1}$,

with $r$ the proton-neutron distance, $u(r)$ and $w(r)$ are the $\mathrm{S}$-wave and D-wave radial wave functions, $Y_{\ell m}$ are spherical harmonics and $\chi_{1}$ the triplet spin wave function, as can be found in nuclear physics textbooks. Since the D-state admixture is small, the (observable) quadrupole moment of the deuteron is mostly proportional to the cross term $\int u w r^{2} d r$, while the D-state probability is proportional to $\int r^{2} w^{2} d r$ and is therefore sensitive to the interior of the wave function, as the centrifugal barrier constricts the D-wave component to the nuclear interior as compared to the S-wave component. It was soon understood. that the D-state probability is not an observable and depends quite strongly on the framework used to describe the deuteron and the cutoff scale in that framework (e.g. see Refs. [81,82]). To summarize in few words, unitary transformations, which leave observable quantities unchanged, modify the wave function of the deuteron in terms of S and D wave components, making them model dependent. The same conclusions apply in principle to the above-discussed orbital occupancies of the halo neutrons in ${ }^{11} \mathrm{Li}$ and any other nucleus, as well as other observables such as momentum distributions $[83,84]$. Such 
non-observable quantities are nevertheless important in our description of nuclear structure, but the model dependence has to be kept in mind when results of different studies are being compared.

Such ambiguities also apply to the interpretation of reaction observables. The electro-disintegration of the deuteron $e+d \rightarrow e+p+n$ can be seen as the simplest breakup reaction sensitive to the internal structure of a nucleus: the involved bound nuclei are reduced to the deuteron itself in the entrance channel and the electro-disintegration process relies on the electromagnetic interaction.

First studies of electro-disintegration of the deuteron were performed at rather low incident energy and low momentum transfer, below $300 \mathrm{MeV} / \mathrm{c}$ [85-87]. At such low momentum transfer, the interpretation of the data required to take into account final state interactions (FSI) $[88,89]$ and mesonexchange currents (MEC). Later measurements at Saclay [90,91], with a momentum transfer between 300 and 700 $\mathrm{MeV} / \mathrm{c}$, and at CEBAF [92] allowed to minimize these effects of FSI and EMC as the momentum transfer $Q^{2}$ of the virtual photon was increased [93]. Our understanding of the structure of the deuteron, and the high momentum component of the wave function, started from assuming a well controlled reaction mechanism and the plane wave approximation. The additional precision data in different kinematic regions (incident energy, momentum and energy transfer) shifted the focus of research from structure only to both the structure of the deuteron and the electron-scattering reaction mechanism (see [94] for a brief overview).

More recently, the electrodisintegration of the deuteron was used as a controlled laboratory to study how the factorization between reaction and structure components of the cross section is modified through renormalization-group transformations [95]. In particular, the scale dependence of the factorisation between the reaction and structure components of the cross section is well illustrated by a first-order analytical calculation. Assuming a unitary transform operator $U(\lambda)$, as discussed in Section 2, any operator is evolved as

$$
\widehat{O}^{\lambda} \equiv U \widehat{O} U^{\dagger}=\widehat{O}+\widetilde{U} \widehat{O}-\widehat{O} \widetilde{U}+\mathscr{O}\left(\widetilde{U}^{2}\right)
$$

where $\widetilde{U}$ is the smooth part of the unitary transform, residual of the identity operator $I$ and defined as $U=I+\widetilde{U}$. With the above, the evolved matrix element that enters the cross section is given by

$$
\begin{aligned}
\left\langle\Psi_{f}^{\lambda}\left|\widehat{O}^{\lambda}\right| \Psi_{i}^{\lambda}\right\rangle= & \left\langle\Psi_{f}|\widehat{O}| \Psi_{i}\right\rangle+\underbrace{\left\langle\Psi_{f}|\widetilde{U} \widehat{O}-\widehat{O} \widetilde{U}| \Psi_{i}\right\rangle}_{\delta \widehat{O}} \\
& +\underbrace{\left\langle\Psi_{f}|\widehat{O} \widetilde{U}| \Psi_{i}\right\rangle}_{\delta\left|\Psi_{i}^{\lambda}\right\rangle}-\underbrace{\left\langle\Psi_{f}|\widetilde{U} \widehat{O}| \Psi_{i}\right\rangle}_{\delta\left\langle\Psi_{f}^{\lambda}\right|} .
\end{aligned}
$$

From Eq. 14, it is clear that the unitary transform does not change the observable, while the evolution of the operator modifies the reaction amplitude (first term of the right hand side) by $\delta \hat{O}$ (second term) which is reshuffled into a modification of the wave functions from modified initial and final state interactions, $\delta\left|\Psi_{i}^{\lambda}\right\rangle$ and $\delta\left|\Psi_{f}^{\lambda}\right\rangle$ (third and fourth terms), respectively.

While the results further demonstrate that scale dependence needs to be taken into account in low-energy nuclear physics, the RG methods show promise to be applicable to breakup reactions in the mid-term future. With the development of modern radioactive-ion beam facilities worldwide, allowing the production of intense beams at various energies and the impressive development of nuclear structure theory over the past few decades, it is foreseeable that the interest in the details of nuclear reaction mechanisms as a tool to extract nuclear structure will grow and develop more and more as a topic of study, as was the case for electron-induced deuteron studies over more than 50 years.

\section{Conclusion}

In this article, we have reviewed the sources of ambiguities in the interpretation of breakup reaction cross sections through two extensively-studied nuclear systems, the twoneutron halo ${ }^{11} \mathrm{Li}$ and the stable deuteron. These ambiguities are associated to the description of the reaction mechanisms and to the inherently non-observable character of certain quantities, shell occupancies in the above examples, used to interpret the data.

The exciting case of ${ }^{11} \mathrm{Li}$ was used to illustrate ambiguities related to the reaction mechanism. Typical variations of $10 \%$ on the $s, p$ and $d$ wave components for the two halo neutrons are encountered.

The electro-disintegration of the deuteron, despite its apparent simplicity, has been used as a benchmark to illustrate how that the factorization of the reaction mechanism and structure depends on the scheme and momentum scale of the theoretical framework used for the description of the data [95]. Our interpretation of reaction observables in terms of nuclear-structure non-observable quantities, such as the deuteron $d$-state probability, is therefore scale and scheme dependent as well. For the deuteron $d$-state probability this ambiguity in the description results in a range of values from 4 to $8 \%$ depending on the chosen $\mathrm{NN}$ interaction and its highmomentum behaviour. Similar scale-dependence on shelloccupancies and spectroscopic factors exist on nucleonremoval reactions with stable and unstable nuclei, and can lead to ambiguities if the framework and momentum scale of the model are not established.

The selection of the scale of the model is a formidable challenge, since so far the description of the reaction observ- 
ables has implied different descriptions with different frameworks and momentum scales for the reaction mechanism and the structure of the involved nuclei. Despite these inconsistencies, the standard approach to the description of nuclear breakup reactions (as described in Sect. 2) has provided remarkable advancements in our understanding of nuclear structure and reactions. There are still discrepancies between the experiment and predictions, in particular in the last decades with radioactive nuclei. These discrepancies, e.g. the isospin dependence of the nucleon removal cross sections, are being investigated to reduce the ambiguities, i.e., here a potential systematic bias in the reaction modeling, in the interpretation of breakup reaction observables.

Finally, we conclude on the words of C. Bertulani and M. Hussein that triggered this contribution: due to the limited experimental information about very exotic nuclei, the interpretation of recent experimental studies often remain ambiguous. The ambiguities can be reduced by confronting nuclear-structure interpretations from different measurements, i.e., different observables. The modeling of nuclear breakup reactions still leads to uncertainties in the interpretation of reaction observables in terms of nuclear structure. In the case of deeply-bound nucleon removal, the interpretation in terms of spectroscopic factors can vary up to a factor of 4 depending on the reaction and models used. Still, another ambiguity arises from the scale and scheme of the theoretical framework when not well defined. A consistent treatment of reaction and structure is therefore a high-priority target of upcoming reaction-theory developments in order to eliminate this ambiguity.

Acknowledgements The authors are thankful to J. Casal for providing the integrated ${ }^{11} \mathrm{Li}$ wavefunction used for Fig. 1 and to Y. Kubota for providing Table 1. Support from the Alexander von Humboldt foundation is acknowledged. This work has been performed in the context of the Grant SFB1245 from the Deutsche Forschungsgemeinschaft (DFG, German Research Foundation).

Funding Information Open Access funding enabled and organized by Projekt DEAL.

Data Availability Statement This manuscript has no associated data or the data will not be deposited. [Authors' comment: This work partly relies on published data. No new data are presented.]

Open Access This article is licensed under a Creative Commons Attribution 4.0 International License, which permits use, sharing, adaptation, distribution and reproduction in any medium or format, as long as you give appropriate credit to the original author(s) and the source, provide a link to the Creative Commons licence, and indicate if changes were made. The images or other third party material in this article are included in the article's Creative Commons licence, unless indicated otherwise in a credit line to the material. If material is not included in the article's Creative Commons licence and your intended use is not permitted by statutory regulation or exceeds the permitted use, you will need to obtain permission directly from the copyright holder. To view a copy of this licence, visit http://creativecomm ons.org/licenses/by/4.0/.

\section{References}

1. I. Tanihata, H. Hamagaki, O. Hashimoto, S. Nagamiya, Y. Shida, N. Yoshikawa, O. Yamakawa, K. Sugimoto, T. Kobayashi, D.E. Greiner, N. Takahashi, Y. Nojiri, Measurements of interaction cross sections and radii of he isotopes. Phys. Lett. B 160(6), 380-384 (1985)

2. I. Tanihata, H. Hamagaki, O. Hashimoto, Y. Shida, N. Yoshikawa, K. Sugimoto, O. Yamakawa, T. Kobayashi, N. Takahashi, Measurements of interaction cross sections and nuclear radii in the light $p$-shell region. Phys. Rev. Lett. 55, 2676-2679 (1985)

3. W. Mittig, J .M. Chouvel, Zhan Wen Long, L. Bianchi, A. Cunsolo, B. Fernandez, A. Foti, J. Gastebois, A. Gillibert, C. Gregoire, Y. Schutz, C. Stephan, Measurement of total reaction cross sections of exotic neutron-rich nuclei. Phys. Rev. Lett. 59, 1889-1891 (1987)

4. I. Tanihata, T. Kobayashi, O. Yamakawa, S. Shimoura, K. Ekuni, K. Sugimoto, N. Takahashi, T. Shimoda, H. Sato, Measurement of interaction cross sections using isotope beams of be and $\mathrm{b}$ and isospin dependence of the nuclear radii. Phys. Lett. B 206(4), 592596 (1988)

5. M.G. Saint-Laurent, R. Anne, D. Bazin, D. Guillemaud-Mueller, U. Jahnke, J.G. Ming, A.C. Mueller, J.F. Bruandet, F. Glasser, S. Kox, E. Liatard, T.U. Chan, G.J. Costa, C. Heitz, Y. El-Masri, F. Hanappe, R. Bimbot, E. Arnold, R. Neugart, Total cross sections of reactions induced by neutron-rich light nuclei. Zeitschrift für Physik A Atomic Nuclei 332(4), 457-465 (1989)

6. C.A. Bertulani, M.S. Hussein, Direct versus sequential fragmentation of neutron-rich nuclei. Phys. Rev. Lett. 64, 1099-1102 (1990)

7. R.J. Furnstahl, H.-W. Hammer, Are occupation numbers observable? Phys. Lett. B 531(3), 203-208 (2002)

8. T. Duguet, G. Hagen, Ab initio approach to effective single-particle energies in doubly closed shell nuclei. Phys. Rev. C 85 (2012)

9. A.M. Mukhamedzhanov, A.S. Kadyrov, Unitary correlation in nuclear reaction theory: separation of nuclear reactions and spectroscopic factors. Phys. Rev. C 82 (2010)

10. T. Duguet, H. Hergert, J.D. Holt, V. Somà, Nonobservable nature of the nuclear shell structure: Meaning, illustrations, and consequences. Phys. Rev. C 92 (2015)

11. T. Kobayashi, O. Yamakawa, K. Omata, K. Sugimoto, T. Shimoda, N. Takahashi, I. Tanihata, Projectile fragmentation of the extremely neutron-rich nucleus ${ }^{11} \mathrm{Li}$ at 0.79 gev/nucleon. Phys. Rev. Lett. 60, 2599-2602 (1988)

12. T. Kobayashi, S. Shimoura, I. Tanihata, K. Katori, K. Matsuta, T. Minamisono, K. Sugimoto, W. Müller, D.L. Olson, T.J.M. Symons, H. Wieman, Electromagnetic dissociation and soft giant dipole resonance of the neutron-dripline nucleus 11li. Phys. Lett. B 232(1), 51-55 (1989)

13. F. Humbert, T. Nilsson, W. Schwab, M. Zinser, Th Blaich, M.J.G. Borge, L.V. Chulkov, ThW Elze, H. Emling, B. Franzke, H. Freiesleben, H. Geissel, K. Grimm, D. Guillemaud-Mueller, P.G. Hansen, R. Holzmann, H. Irnich, L. Johannsen, B. Jonson, J.G. Keller, O. Klepper, H. Klingler, J.V. Kratz, R. Kulessa, D. Lambrecht, Y. Leifels, A. Magel, M. Mohar, A.C. Mueller, G. Münzenberg, P. Møller, F. Nickel, G. Nyman, A. Richter, K. Riisager, C. Scheidenberger, G. Schrieder, B.M. Sherrill, H. Simon, K. Stelzer, J. Stroth, O. Tengblad, W. Trautmann, E. Wajda, E. Zude, Longitudinal and transverse momentum distributions of 91i fragments from break-up of 11li. Phys. Lett. B 347(3), 198-204 (1995)

14. N.A. Orr, N. Anantaraman, S.M. Austin, C.A. Bertulani, K. Hanold, J.H. Kelley, D.J. Morrissey, B.M. Sherrill, G.A. Souliotis, M. Thoennessen, J.S. Winfield, J.A. Winger, Momentum distributions of ${ }^{9} \mathrm{Li}$ fragments following the breakup of ${ }^{11} \mathrm{Li}$. Phys. Rev. Lett. 69, 2050-2053 (1992)

15. K. Ieki, D. Sackett, A. Galonsky, C.A. Bertulani, J.J. Kruse, W.G. Lynch, D.J. Morrissey, N.A. Orr, H. Schulz, B.M. Sherrill, A. Sus- 
tich, J.A. Winger, F. Deák, Á. Horváth, Á. Kiss, Z. Seres, J.J. Kolata, R.E. Warner, D.L. Humphrey, Coulomb dissociation of ${ }^{11}$ Li. Phys. Rev. Lett. 70, 730-733 (1993)

16. Carlos A Bertulani, LFelipe Canto, Mahir S Hussein, The structure and reactions of neutron-rich nuclei. Phys. Rep. 226(6), 281-376 (1993)

17. M.V.Zhukov, B.V. Danilin, D.V. Fedorov, J.S. Vaagen, F.A. Gareev, J. Bang, Calculation of 11 li in the framework of a three-body model with simple central potentials. Phys. Lett. B 265(1), 19-22 (1991)

18. M.V. Zhukov, D.V. Fedorov, B.V. Danilin, J.S. Vaagen, J.M. Bang, A simplified three-body model for 11li and associated 9li and neutron momentum distributions. Nucl. Phys. A 529(1), 53-67 (1991)

19. E. Garrido, D.V. Fedorov, A.S. Jensen, Breakup reactions of ${ }^{11} \mathrm{Li}$ within a three-body model. Phys. Rev. C 59, 1272-1289 (1999)

20. H. Esbensen, G.F. Bertsch, K. Ieki, Momentum distributions for $\left({ }^{11} \mathrm{li},{ }^{9} \mathrm{Li}+\mathrm{n}+\mathrm{n}\right)$ breakup reactions. Phys. Rev. C 48, 326-331 (1993)

21. L.A. Souza, T. Frederico, C.A. Bertulani, Core longitudinal momentum distributions in the stripping reactions of two-neutron halo nuclei. J. Phys. Conf. Ser. 1291 (2019)

22. M.S. Hussein, K.W. McVoy, Inclusive projectile fragmentation in the spectator model. Nucl. Phys. A 445(1), 124-139 (1985)

23. C.A. Bertulani, K.W. McVoy, Momentum distributions in reactions with radioactive beams. Phys. Rev. C 46, 2638-2641 (1992)

24. J. Casal, M. Gómez-Ramos, A.M. Moro, Description of the 11li(p, d)10li transfer reaction using structure overlaps from a full threebody model. Phys. Lett. B 767, 307-313 (2017)

25. M. Gómez-Ramos, J. Casal, A.M. Moro, Linking structure and dynamics in (p, pn) reactions with Borromean nuclei: the 11li(p, pn) 10 li case. Phys. Lett. B 772, 115-120 (2017)

26. B Alex Brown, New skyrme interaction for normal and exotic nuclei. Phys. Rev. C 58, 220-231 (1998)

27. Isao Tanihata, Herve Savajols, Rituparna Kanungo, Recent experimental progress in nuclear halo structure studies. Prog. Part. Nucl. Phys. 68, 215-313 (2013)

28. Y. Aksyutina, H.T. Johansson, P. Adrich, F. Aksouh, T. Aumann, K. Boretzky, M.J.G. Borge, A. Chatillon, L.V. Chulkov, D. CortinaGil, U. Datta Pramanik, H. Emling, C. Forssén, H.O.U. Fynbo, H. Geissel, M. Hellström, G. Ickert, K.L. Jones, B. Jonson, A. Kliemkiewicz, J.V. Kratz, R. Kulessa, M. Lantz, T. LeBleis, A.O. Lindahl, K. Mahata, M. Matos, M. Meister, G. Münzenberg, T. Nilsson, G. Nyman, R. Palit, M. Pantea, S. Paschalis, W. Prokopowicz, R. Reifarth, A. Richter, K. Riisager, G. Schrieder, H. Simon, K. Sümmerer, O. Tengblad, W. Walus, H. Weick, M.V. Zhukov, Lithium isotopes beyond the drip line. Phys. Lett. B 666(5), 430 - 434 (2008)

29. Y. Aksyutina, T. Aumann, K. Boretzky, M.J.G. Borge, C. Caesar, A. Chatillon, L.V. Chulkov, D. Cortina-Gil, U. Datta Pramanik, H. Emling, H.O.U. Fynbo, H. Geissel, G. Ickert, H.T. Johansson, B. Jonson, R. Kulessa, C. Langer, T. LeBleis, K. Mahata, G. Münzenberg, T. Nilsson, G. Nyman, R. Palit, S. Paschalis, W. Prokopowicz, R. Reifarth, D. Rossi, A. Richter, K. Riisager, G. Schrieder, H. Simon, K. Sümmerer, O. Tengblad, H. Weick, M.V. Zhukov, Momentum profile analysis in one-neutron knockout from Borromean nuclei. Phys. Lett. B 718(4), 1309-1313 (2013)

30. A.M. Moro, Three-body model for the analysis of quasifree scattering reactions in inverse kinematics. Phys. Rev. C 92 (2015)

31. H. Simon, M. Meister, T. Aumann, M.J.G. Borge, L.V. Chulkov, U. Datta Pramanik, Th.W. Elze, H. Emling, C. Forssén, H. Geissel, M. Hellström, B. Jonson, J.V. Kratz, R. Kulessa, Y. Leifels, K. Markenroth, G. Münzenberg, F. Nickel, T. Nilsson, G. Nyman, A. Richter, K. Riisager, C. Scheidenberger, G. Schrieder, O. Tengblad, M.V. Zhukov. Systematic investigation of the drip-line nuclei $11 \mathrm{li}$ and 14be and their unbound subsystems 10li and 13be. Nucl. Phys. A 791(3), 267-302 (2007)
32. A.M. Moro, J. Casal, M. Gómez-Ramos, Investigating the $101 \mathrm{i}$ continuum through 9li(d, p)10li reactions. Phys. Lett. B 793, 1318 (2019)

33. B.V. Carlson, T. Frederico, M.S. Hussein, Inclusive breakup of three-fragment weakly bound nuclei. Phys. Lett. B 767, 53-57 (2017)

34. F.M. Marqués, M. Labiche, N.A. Orr, J. C. Angélique, L. Axelsson, B. Benoit, U.C. Bergmann, M.J.G. Borge, W.N. Catford, S.P.G. Chappell, N.M. Clarke, G. Costa, N. Curtis, A. D'Arrigo, E. de Góes Brennand, F. de Oliveira Santos, O. Dorvaux, G. Fazio, M. Freer, B.R. Fulton, G. Giardina, S. Grévy, D. Guillemaud-Mueller, F. Hanappe, B. Heusch, B. Jonson, C. Le Brun, S. Leenhardt, M. Lewitowicz, M.J. López, K. Markenroth, A.C. Mueller, T. Nilsson, A. Ninane, G. Nyman, I. Piqueras, K. Riisager, M.G. Saint Laurent, F. Sarazin, S. M. Singer, O. Sorlin, L. Stuttgé, Three-body correlations in Borromean halo nuclei. Phys. Rev. C 64, 061301 (2001)

35. I. Tanihata, T. Kobayashi, T. Suzuki, K. Yoshida, S. Shimoura, K. Sugimoto, K. Matsuta, T. Minamisono, W. Christie, D. Olson, H. Wieman, Determination of the density distribution and the correlation of halo neutrons in 11li. Phys. Lett. B 287(4), 307-311 (1992)

36. K. Ieki, A. Galonsky, D. Sackett, J.J. Kruse, W.G. Lynch, D.J. Morrissey, N.A. Orr, B.M. Sherrill, J.A. Winger, F. Deák, Á. Horváth, Á. Kiss, Z. Seres, J.J. Kolata, R.E. Warner, D.L. Humphrey, Is there a bound dineutron in ${ }^{11}$ ?. Phys. Rev. C 54, 1589-1591 (1996)

37. M. Zinser, F. Humbert, T. Nilsson, W. Schwab, H. Simon, T. Aumann, M.J.G. Borge, L.V. Chulkov, J. Cub, ThW Elze, H. Emling, H. Geissel, D. Guillemaud-Mueller, P.G. Hansen, R. Holzmann, H. Irnich, B. Jonson, J.V. Kratz, R. Kulessa, Y. Leifels, H. Lenske, A. Magel, A.C. Mueller, G. Münzenberg, F. Nickel, G. Nyman, A. Richter, K. Riisager, C. Scheidenberger, G. Schrieder, K. Stelzer, J. Stroth, A. Surowiec, O. Tengblad, E. Wajda, E. Zude, Invariant-mass spectroscopy of 10li and 11li. Nucl. Phys. A 619(1), 151-176 (1997)

38. T. Nakamura, A.M. Vinodkumar, T. Sugimoto, N. Aoi, H. Baba, D. Bazin, N. Fukuda, T. Gomi, H. Hasegawa, N. Imai, M. Ishihara, T. Kobayashi, Y. Kondo, T. Kubo, M. Miura, T. Motobayashi, H. Otsu, A. Saito, H. Sakurai, S. Shimoura, K. Watanabe, Y.X. Watanabe, T. Yakushiji, Y. Yanagisawa, K. Yoneda, Observation of strong lowlying $e 1$ strength in the two-neutron halo nucleus ${ }^{11} \mathrm{Li}$. Phys. Rev. Lett. 96 (2006)

39. S. Shimoura, T. Nakamura, M. Ishihara, N. Inabe, T. Kobayashi, T. Kubo, R.H. Siemssen, I. Tanihata, Y. Watanabe, Coulomb dissociation reaction and correlations of two halo neutrons in 11li. Phys. Lett. B 348(1), 29-34 (1995)

40. H. Simon, D. Aleksandrov, T. Aumann, L. Axelsson, T. Baumann, M.J.G. Borge, L.V. Chulkov, R. Collatz, J. Cub, W. Dostal, B. Eberlein, ThW Elze, H. Emling, H. Geissel, A. Grünschloss, M. Hellström, J. Holeczek, R. Holzmann, B. Jonson, J.V. Kratz, G. Kraus, R. Kulessa, Y. Leifels, A. Leistenschneider, T. Leth, I. Mukha, G. Münzenberg, F. Nickel, T. Nilsson, G. Nyman, B. Petersen, M. Pfützner, A. Richter, K. Riisager, C. Scheidenberger, G. Schrieder, W. Schwab, M.H. Smedberg, J. Stroth, A. Surowiec, O. Tengblad, M.V.Zhukov, Direct experimental evidence for strong admixture of different parity states in ${ }^{11}$ Li. Phys. Rev. Lett. 83, 496-499 (1999)

41. Y. Kubota, A. Corsi, G. Authelet, H. Baba, C. Caesar, D. Calvet, A. Delbart, M. Dozono, J. Feng, F. Flavigny, J.-M. Gheller, J. Gibelin, A. Giganon, A. Gillibert, K. Hasegawa, T. Isobe, Y. Kanaya, S. Kawakami, D. Kim, Y. Kikuchi, Y. Kiyokawa, M. Kobayashi, N. Kobayashi, T. Kobayashi, Y. Kondo, Z. Korkulu, S. Koyama, V. Lapoux, Y. Maeda, F.M. Marqués, T. Motobayashi, T. Miyazaki, T. Nakamura, N. Nakatsuka, Y. Nishio, A. Obertelli, K. Ogata, A. Ohkura, N.A. Orr, S. Ota, H. Otsu, T. Ozaki, V. Panin, S. Paschalis, E.C. Pollacco, S. Reichert, J.-Y. Roussé, A.T. Saito, S. Sakaguchi, M. Sako, C. Santamaria, M. Sasano, H. Sato, M. Shikata, Y. Shimizu, Y. Shindo, L. Stuhl, T. Sumikama, Y.L. Sun, 
M. Tabata, Y. Togano, J. Tsubota, Z.H. Yang, J. Yasuda, K. Yoneda, J.Zenihiro, T. Uesaka, Surface localization of the dineutron in ${ }^{11} \mathrm{Li}$. Phys. Rev. Lett. 125 (2020)

42. N.B. Shulgina, B. Jonson, M.V. Zhukov, 11li structure from experimental data. Nucl. Phys. A 825(3-4), 175-199 (2009)

43. A. Sanetullaev, R. Kanungo, J. Tanaka, M. Alcorta, C. Andreoiu, P. Bender, A.A. Chen, G. Christian, B. Davids, J. Fallis, J.P. Fortin, N. Galinski, A.T. Gallant, P.E. Garrett, G. Hackman, B. Hadinia, S. Ishimoto, M. Keefe, R. Krücken, J. Lighthall, E. McNeice, D. Miller, J. Purcell, J.S. Randhawa, T. Roger, A. Rojas, H. Savajols, A. Shotter, I. Tanihata, I.J. Thompson, C. Unsworth, P. Voss, $\mathrm{Z}$. Wang, Investigation of the role of 10li resonances in the halo structure of 11li through the transfer reaction. Phys. Lett. B 755, 481-485 (2016)

44. I. Tanihata, M. Alcorta, D. Bandyopadhyay, R. Bieri, L. Buchmann, B. Davids, N. Galinski, D. Howell, W. Mills, S. Mythili, R. Openshaw, E. Padilla-Rodal, G. Ruprecht, G. Sheffer, A.C. Shotter, M. Trinczek, P. Walden, H. Savajols, T. Roger, M. Caamano, W. Mittig, P. Roussel-Chomaz, R. Kanungo, A. Gallant, M. Notani, G. Savard, I.J. Thompson, Measurement of the two-halo neutron transfer reaction ${ }^{1} \mathrm{H}\left({ }^{11} \mathrm{Li},{ }^{9} \mathrm{Li}\right){ }^{3} \mathrm{H}$ at $3 a \mathrm{MeV}$. Phys. Rev. Lett. 100 (2008)

45. K. Hagino, H. Sagawa, Pairing correlations in nuclei on the neutron-drip line. Phys. Rev. C 72 (2005)

46. Y. Kikuchi, T. Myo, K. Katō, K. Ikeda, Coulomb breakup reactions of ${ }^{11}$ li in the coupled-channel ${ }^{9} \mathrm{li}+n+n$ model. Phys. Rev. C 87 (2013)

47. K. Ikeda, T. Myo, K. Kato, H. Toki, Clusters in Nuclei: Volume 1, Di-Neutron Clustering and Deuteron-like Tensor Correlation in Nuclear Structure Focusing on ${ }^{11} \mathrm{Li}$, pp. 165-221. Springer, Berlin (2010)

48. M.H. Macfarlane, J.B. French, Stripping reactions and the structure of light and intermediate nuclei. Rev. Mod. Phys. 32, 567-691 (1960)

49. P.G. Hansen, J.A. Tostevin, Direct reactions with exotic nuclei. Annu. Rev. Nucl. Part. Sci. 53(1), 219-261 (2003)

50. A. Gade, P. Adrich, D. Bazin, M.D. Bowen, B.A. Brown, C.M. Campbell, J.M. Cook, T. Glasmacher, P.G. Hansen, K. Hosier, S. McDaniel, D. McGlinchery, A. Obertelli, K. Siwek, L.A. Riley, J.A. Tostevin, D. Weisshaar, Reduction of spectroscopic strength: weakly-bound and strongly-bound single-particle states studied using one-nucleon knockout reactions. Phys. Rev. C 77 (2008)

51. J.A. Tostevin, A. Gade, Systematics of intermediate-energy singlenucleon removal cross sections. Phys. Rev. C 90 (2014)

52. J. Lee, M.B. Tsang, D. Bazin, D. Coupland, V. Henzl, D. Henzlova, M. Kilburn, W.G. Lynch, A.M. Rogers, A. Sanetullaev, A. Signoracci, Z.Y. Sun, M. Youngs, K.Y. Chae, R.J. Charity, H.K. Cheung, M. Famiano, S. Hudan, P. O'Malley, W.A. Peters, K. Schmitt, D. Shapira, L.G. Sobotka, Neutron-proton asymmetry dependence of spectroscopic factors in ar isotopes. Phys. Rev. Lett. 104, 112701 (2010)

53. F. Flavigny, A. Gillibert, L. Nalpas, A. Obertelli, N. Keeley, C. Barbieri, D. Beaumel, S. Boissinot, G. Burgunder, A. Cipollone, A. Corsi, J. Gibelin, S. Giron, J. Guillot, F. Hammache, V. Lapoux, A. Matta, E.C. Pollacco, R. Raabe, M. Rejmund, N. de Séreville, A. Shrivastava, A. Signoracci, Y. Utsuno, Limited asymmetry dependence of correlations from single nucleon transfer. Phys. Rev. Lett. 110 (2013)

54. B.P. Kay, J.P. Schiffer, S.J. Freeman, Quenching of cross sections in nucleon transfer reactions. Phys. Rev. Lett. 111 (2013)

55. Y.P. Xu, D.Y. Pang, X.Y. Yun, C. Wen, C.X. Yuan, J.L. Lou, Proton-neutron asymmetry independence of reduced singleparticle strengths derived from (p, d) reactions. Phys. Lett. B 790, 308-313 (2019)

56. T. Aumann, C. Barbieri, D. Bazin, C.A. Bertulani, A. Bonaccorso, W.H. Dickhoff, A. Gade, M. Gómez-Ramos, B.P. Kay, A.M. Moro,
T. Nakamura, A. Obertelli, K. Ogata, S. Paschalis, T. Uesaka, Quenching of single-particle strength from direct reactions with stable and rare-isotope beams. Prog. Part. Nucl. Phys. (2020)

57. D. Bazin, R.J. Charity, R.T. de Souza, M.A. Famiano, A. Gade, V. Henzl, D. Henzlova, S. Hudan, J. Lee, S. Lukyanov, W.G. Lynch, S. McDaniel, M. Mocko, A. Obertelli, A.M. Rogers, L.G. Sobotka, J.R. Terry, J.A. Tostevin, M.B. Tsang, M.S. Wallace, Mechanisms in knockout reactions. Phys. Rev. Lett. 102 (2009)

58. K. Wimmer, D. Bazin, A. Gade, J.A. Tostevin, T. Baugher, Z. Chajecki, D. Coupland, M.A. Famiano, T.K. Ghosh, G.F. Grinyer, M.E. Howard, M. Kilburn, W.G. Lynch, B. Manning, K. Meierbachtol, P. Quarterman, A. Ratkiewicz, A. Sanetullaev, R.H. Showalter, S.R. Stroberg, M.B. Tsang, D. Weisshaar, J. Winkelbauer, R. Winkler, M. Youngs, Elastic breakup cross sections of well-bound nucleons. Phys. Rev. C 90 (2014)

59. J. Hüfner, M.C. Nemes, Relativistic heavy ions measure the momentum distribution on the nuclear surface. Phys. Rev. C 23, 2538-2547 (1981)

60. J.A. Tostevin, Single-nucleon knockout reactions at fragmentation beam energies. Nucl. Phys. A 682(1), 320-331 (2001)

61. P.G. Hansen, Momentum content of single-nucleon halos. Phys. Rev. Lett. 77, 1016-1019 (1996)

62. J.S. Al-Khalili, Validity of spectator core model in neutron knockout reactions. Nucl. Phys. A 689(1), 551-554 (2001) (European Few-Body Problems in Physics)

63. C. Louchart, A. Obertelli, A. Boudard, F. Flavigny, Nucleon removal from unstable nuclei investigated via intranuclear cascade. Phys. Rev. C 83 (2011)

64. Y.L. Sun, J. Lee, Y.L. Ye, A. Obertelli, Z.H. Li, N. Aoi, H.J. Ong, Y. Ayyad, C.A. Bertulani, J. Chen, A. Corsi, F. Cappuzzello, M. Cavallaro, T. Furono, Y.C. Ge, T. Hashimoto, E. Ideguchi, T. Kawabata, J.L. Lou, Q.T. Li, G. Lorusso, F. Lu, H.N. Liu, S. Nishimura, H. Suzuki, J. Tanaka, M. Tanaka, D.T. Tran, M.B. Tsang, J. Wu, Z.Y. $\mathrm{Xu}, \mathrm{T}$. Yamamoto, Experimental study of the knockout reaction mechanism using ${ }^{14} o$ at 60 mev/nucleon. Phys. Rev. C 93 (2016)

65. K. Ogata, K. Yoshida, K. Minomo, Asymmetry of the parallel momentum distribution of ( $p, p n)$ reaction residues. Phys. Rev. C 92 (2015)

66. A. Gade, D. Bazin, C.A. Bertulani, B.A. Brown, C.M. Campbell, J.A. Church, D.C. Dinca, J. Enders, T. Glasmacher, P.G. Hansen, Z. Hu, K.W. Kemper, W.F. Mueller, H. Olliver, B.C. Perry, L.A. Riley, B.T. Roeder, B.M. Sherrill, J.R. Terry, J.A. Tostevin, K.L. Yurkewicz, Knockout from ${ }^{46} \mathrm{Ar}: \ell=3$ neutron removal and deviations from eikonal theory. Phys. Rev. C 71 (2005)

67. K.L. Yurkewicz, D. Bazin, B.A. Brown, J. Enders, A. Gade, T. Glasmacher, P.G. Hansen, V. Maddalena, A. Navin, B.M. Sherrill, J.A. Tostevin, One-neutron knockout from ${ }^{57} \mathrm{Ni}$. Phys. Rev. C 74 (2006)

68. F. Flavigny, A. Obertelli, A. Bonaccorso, G.F. Grinyer, C. Louchart, L. Nalpas, A. Signoracci, Nonsudden limits of heavy-ion induced knockout reactions. Phys. Rev. Lett. 108 (2012)

69. J.A. Tostevin, D. Bazin, B.A. Brown, T. Glasmacher, P.G. Hansen, V. Maddalena, A. Navin, B.M. Sherrill, Single-neutron removal reactions from ${ }^{15} \mathrm{C}$ and ${ }^{11} \mathrm{Be}$ : deviations from the eikonal approximation. Phys. Rev. C 66 (2002)

70. A. Bonaccorso, D.M. Brink, Nucleon transfer to continuum states. Phys. Rev. C 38, 1776-1786 (1988)

71. A. Bonaccorso, D.M. Brink, Stripping to the continuum of ${ }^{208} \mathrm{~Pb}$. Phys. Rev. C 44, 1559-1568 (1991)

72. S. Kawase, T. Uesaka, T. Leung Tang, D. Beaumel, M. Dozono, T. Fukunaga, T. Fujii, N. Fukuda, A. Galindo-Uribarri, S. Hwang, N. Inabe, T. Kawabata, T. Kawahara, W. Kim, K. Kisamori, M. Kobayashi, T. Kubo, Y. Kubota, K. Kusaka, C. Lee, Y. Maeda, H. Matsubara, S. Michimasa, H. Miya, T. Noro, Y. Nozawa, A. Obertelli, K. Ogata, S. Ota, E. Padilla-Rodal, S. Sakaguchi, H. Sakai, M. Sasano, S. Shimoura, S. Stepanyan, H. Suzuki, T. Suzuki, 
M. Takaki, H. Takeda, A. Tamii, H. Tokieda, T. Wakasa, T. Wakui, K. Yako, J. Yasuda, Y. Yanagisawa, R. Yokoyama, K. Yoshida, K. Yoshida, J. Zenihiro, Exclusive quasi-free proton knockout from oxygen isotopes at intermediate energies. Prog. Theor. Exp. Phys. 2018(2), $021 \mathrm{D} 01$ (2018)

73. L. Atar, S. Paschalis, C. Barbieri, C. A. Bertulani, P. Díaz Fernández, M. Holl, M.A. Najafi, V. Panin, H. Alvarez-Pol, T. Aumann, V. Avdeichikov, S. Beceiro-Novo, D. Bemmerer, J. Benlliure, J.M. Boillos, K. Boretzky, M.J.G. Borge, M. Caamaño, C. Caesar, E. Casarejos, W. Catford, J. Cederkall, M. Chartier, L. Chulkov, D. Cortina-Gil, E. Cravo, R. Crespo, I. Dillmann, Z. Elekes, J. Enders, O. Ershova, A. Estrade, F. Farinon, L.M. Fraile, M. Freer, D. Galaviz Redondo, H. Geissel, R. Gernhäuser, P. Golubev, K. Göbel, J. Hagdahl, T. Heftrich, M. Heil, M. Heine, A. Heinz, A. Henriques, A. Hufnagel, A. Ignatov, H.T. Johansson, B. Jonson, J. Kahlbow, N. Kalantar-Nayestanaki, R. Kanungo, A. KelicHeil, A. Knyazev, T. Kröll, N. Kurz, M. Labiche, C. Langer, T. Le Bleis, R. Lemmon, S. Lindberg, J. Machado, J. MarganiecGałkazka, A. Movsesyan, E. Nacher, E. Y. Nikolskii, T. Nilsson, C. Nociforo, A. Perea, M. Petri, S. Pietri, R. Plag, R. Reifarth, G. Ribeiro, C. Rigollet, D. M. Rossi, M. Röder, D. Savran, H. Scheit, H. Simon, O. Sorlin, I. Syndikus, J.T. Taylor, O. Tengblad, R. Thies, Y. Togano, M. Vandebrouck, P. Velho, V. Volkov, A. Wagner, F. Wamers, H. Weick, C. Wheldon, G.L. Wilson, J.S. Winfield, P. Woods, D. Yakorev, M. Zhukov, A. Zilges, K. Zuber, Quasifree $(p, 2 p)$ reactions on oxygen isotopes: observation of isospin independence of the reduced single-particle strength. Phys. Rev. Lett. 120, 052501 (2018)

74. M. Gómez-Ramos, A.M. Moro, Binding-energy independence of reduced spectroscopic strengths derived from $(\mathrm{p}, 2 \mathrm{p})$ and $(\mathrm{p}, \mathrm{pn})$ reactions with nitrogen and oxygen isotopes. Phys. Lett. B 785, 511-516 (2018)

75. N.T.T. Phuc, K. Yoshida, K. Ogata, Toward a reliable description of $(p, p n)$ reactions in the distorted-wave impulse approximation. Phys. Rev. C 100 (2019)

76. T. Aumann, C.A. Bertulani, J. Ryckebusch, Quasifree $(p, 2 p)$ and $(p, p n)$ reactions with unstable nuclei. Phys. Rev. C 88 (2013)

77. S. Chen, J. Lee, P. Doornenbal, A. Obertelli, C. Barbieri, Y. Chazono, P. Navrátil, K. Ogata, T. Otsuka, F. Raimondi, V. Somà, Y. Utsuno, K. Yoshida, H. Baba, F. Browne, D. Calvet, F. Château, N. Chiga, A. Corsi, M.L. Cortés, A. Delbart, J.-M. Gheller, A. Giganon, A. Gillibert, C. Hilaire, T. Isobe, J. Kahlbow, T. Kobayashi, Y. Kubota, V. Lapoux, H.N. Liu, T. Motobayashi, I. Murray, H. Otsu, V. Panin, N. Paul, W. Rodriguez, H. Sakurai, M. Sasano, D. Steppenbeck, L. Stuhl, Y.L. Sun, Y. Togano, T. Uesaka, K. Wimmer, K. Yoneda, N. Achouri, O. Aktas, T. Aumann, L.X. Chung, F. Flavigny, S. Franchoo, I. Gašparić, R.-B. Gerst, J. Gibelin, K.I. Hahn, D. Kim, T. Koiwai, Y. Kondo, P. Koseoglou, C. Lehr, B.D. Linh, T. Lokotko, M. MacCormick, K. Moschner, T. Nakamura, S.Y. Park, D. Rossi, E. Sahin, D. Sohler, P.-A. Söderström, S. Takeuchi, H. Törnqvist, V. Vaquero, V. Wagner, S. Wang, V. Werner, X. Xu, H. Yamada, D. Yan, Z. Yang, M. Yasuda, L. Zanetti, Quasifree neutron knockout from ${ }^{54} \mathrm{Ca}$ corroborates arising $n=34$ neutron magic number. Phys. Rev. Lett. 123 (2019)

78. Y.L. Sun, A. Obertelli, P. Doornenbal, C. Barbieri, Y. Chazono, T. Duguet, H.N. Liu, P. Navrátil, F. Nowacki, K. Ogata, T. Otsuka, F. Raimondi, V. Somà, Y. Utsuno, K. Yoshida, N. Achouri, H. Baba, F. Browne, D. Calvet, F. Château, S. Chen, N. Chiga, A. Corsi, M.L. Cortés, A. Delbart, J.-M. Gheller, A. Giganon, A. Gillibert, C. Hilaire, T. Isobe, T. Kobayashi, Y. Kubota, V. Lapoux, T. Motobayashi, I. Murray, H. Otsu, V. Panin, N. Paul, W. Rodriguez, H. Sakurai, M. Sasano, D. Steppenbeck, L. Stuhl, Y. Togano, T. Uesaka, K. Wimmer, K. Yoneda, O. Aktas, T. Aumann, L.X. Chung, F. Flavigny, S. Franchoo, I. Gašparić, R.-B. Gerst, J. Gibelin, K.I. Hahn, D. Kim, T. Koiwai, Y. Kondo, P. Koseoglou, J. Lee, C. Lehr, B.D. Linh, T. Lokotko, M. MacCormick, K.
Moschner, T. Nakamura, S.Y. Park, D. Rossi, E. Sahin, D. Sohler, P.-A. Söderström, S. Takeuchi, H. Törnqvist, V. Vaquero, V. Wagner, S. Wang, V. Werner, X. Xu, H. Yamada, D. Yan, Z. Yang, M. Yasuda, L. Zanetti, Restoration of the natural e(1/21+) - e(3/21+) energy splitting in odd-k isotopes towards $n=40$. Phys. Lett. B 802 (2020)

79. V. Panin, J.T. Taylor, S. Paschalis, F. Wamers, Y. Aksyutina, H. Alvarez-Pol, T. Aumann, C.A. Bertulani, K. Boretzky, C. Caesar, M. Chartier, L.V. Chulkov, D. Cortina-Gil, J. Enders, O. Ershova, H. Geissel, R. Gernhäuser, M. Heil, H.T. Johansson, B. Jonson, A. Kelić-Heil, C. Langer, T. Le Bleis, R. Lemmon, T. Nilsson, M. Petri, R. Plag, R. Reifarth, D. Rossi, H. Scheit, H. Simon, H. Weick, C. Wimmer, Exclusive measurements of quasi-free proton scattering reactions in inverse and complete kinematics. Phys. Lett. B 753, 204-210 (2016)

80. A. Frotscher, M. Gómez-Ramos, A. Obertelli, P. Doornenbal, G. Authelet, H. Baba, D. Calvet, F. Château, S. Chen, A. Corsi, A. Delbart, J.-M. Gheller, A. Giganon, A. Gillibert, T. Isobe, V. Lapoux, M. Matsushita, S. Momiyama, T. Motobayashi, M. Niikura, H. Otsu, N. Paul, C. Péron, A. Peyaud, E. C. Pollacco, J.-Y. Roussé, H. Sakurai, C. Santamaria, M. Sasano, Y. Shiga, N. Shimizu, D. Steppenbeck, S. Takeuchi, R. Taniuchi, T. Uesaka, H. Wang, K. Yoneda, T. Ando, T. Arici, A. Blazhev, F. Browne, A.M. Bruce, R. Carroll, L. X. Chung, M. L. Cortés, M. Dewald, B. Ding, Zs. Dombradi, F. Flavigny, S. Franchoo, F. Giacoppo, M. Górska, A. Gottardo, K. Hadyńska-Klek, Z. Korkulu, S. Koyama, Y. Kubota, A. Jungclaus, J. Lee, M. Lettmann, B.D. Linh, J. Liu, Z. Liu, C. Lizarazo, C. Louchart, R. Lozeva, K. Matsui, T. Miyazaki, K. Moschner, S. Nagamine, N. Nakatsuka, C. Nita, S. Nishimura, C. R. Nobs, L. Olivier, S. Ota, Z. Patel, Z. Podolyák, M. Rudigier, E. Sahin, T.Y. Saito, C. Shand, P.-A. Söderström, I.G. Stefan, T. Sumikama, D. Suzuki, R. Orlandi, V. Vaquero, Z. Vajta, V. Werner, K. Wimmer, J. Wu, Z. Xu, Sequential nature of $(p, 3 p)$ two-proton knockout from neutron-rich nuclei. Phys. Rev. Lett. 125, 012501 (2020)

81. R.D. Amado, The deuteron D state revisited. Comments Nucl. Part. Phys. 10(3), 131-136 (1981)

82. T.E.O. Ericson, M. Rosa-Clot, D-state admixture and tensor forces in light nuclei. Annu. Rev. Nucl. Part. Sci. 35(1), 271-294 (1985)

83. E.R. Anderson, S.K. Bogner, R.J. Furnstahl, R.J. Perry, Operator evolution via the similarity renormalization group: The deuteron. Phys. Rev. C 82 (2010)

84. R.J. Furnstahl, A. Schwenk, How should one formulate, extract and interpret 'non-observables' for nuclei? J. Phys. G Nucl. Part. Phys. 37(6) (2010)

85. M. Croissiaux, Electron-proton coincidences in inelastic electrondeuteron scattering. Phys. Rev. 127, 613-616 (1962)

86. P. Bounin, M. Croissiaux, Experiences de coincidences (e, e'p) sur le deuterium. Nucl. Phys. 70(2), 401-414 (1965)

87. G. van der Steenhoven, Deuterium electro-disintegration experiments, in Mesons and Light Nuclei. Few-Body Systems ed. by E. Truhlík, R. Mach, vol. 5 (Springer, Vienna, 1992)

88. I.J. McGee. Electrodisintegration of the deuteron. I. connection between the $n-p-d$ vertex function and the deuteron wave function. Phys. Rev. 158, 1500-1514 (1967)

89. I.J. McGee. Electrodisintegration of the deuteron. II. final-state interactions. Phys. Rev. 161, 1640-1651 (1967)

90. M. Bernheim, A. BussiÃšre, J. Mougey, D. Royer, D. Tarnowski, S. Turck-Chieze, S. Frullani, G.P. Capitani, E. De Sanctis, E. Jans, Momentum distribution of nucleons in the deuteron from the d(e, eâp)n reaction. Nucl. Phys. A 365(3), 349-370 (1981)

91. S. Auffret, J.M. Cavedon, J.C. Clemens, B. Frois, D. Goutte, M. Huet, Ph Leconte, J. Martino, Y. Mizuno, X.H. Phan, S. Platchkov, I. Sick, Magnetic form factor of the deuteron. Phys. Rev. Lett. 54, 649-652 (1985) 
92. P.E. Ulmer, K.A. Aniol, H. Arenhövel, J.-P. Chen, E. Chudakov, D. Crovelli, J.M. Finn, K.G. Fissum, O. Gayou, J. Gomez, J.O. Hansen, C.W. de Jager, S. Jeschonnek, M.K. Jones, M. Kuss, J.J. LeRose, M. Liang, R.A. Lindgren, S. Malov, D. Meekins, R. Michaels, J. Mitchell, C.F. Perdrisat, V. Punjabi, R. Roché, F. Sabatie, A. Saha, R. Suleiman, L. Todor, B.B. Wojtsekhowski, ${ }^{2 \mathrm{H}}\left(e, e^{\prime} p\right) n$ reaction at high recoil momenta. Phys. Rev. Lett. 89 (2002)

93. J.M. Laget, The electro-disintegration of few body systems revisited. Phys. Lett. B 609(1), 49-56 (2005)
94. R. Gilman, Deuteron elastic scattering, photo- and electrodisintegration. Nucl. Phys. A 737, 156-164 (2004)

95. S.N. More, S. König, R.J. Furnstahl, K. Hebeler, Deuteron electrodisintegration with unitarily evolved potentials. Phys. Rev. C 92 (2015) 